\title{
Sleep disorders in the elderly
}

\section{Christopher E. Jagus \& Susan M. Benbow}

Sleep disorders are common among elderly people, affecting about $50 \%$ compared with about $26 \%$ of younger adults. About two-thirds of residents in long-term care have sleep disturbance (Karacan \& Williams, 1983; Becker \& Jamieson, 1992). This high prevalence of sleep disorders results in excessive daytime napping, fatigue, cognitive impairment, increased psychiatric morbidity and increased prescribing of hypnotics, with an associated increase in mortality (Kripke et al, 1979). Karacan \& Williams (1983) quote figures varying from 26 to $100 \%$ for the prescribing of hypnotics to residents in institutional care. This has major implications. There is a risk of both accidental and deliberate overdose. There may be interactions with other drugs and a hangover effect can impair daytime functioning. Excessive sedation can make the patient difficult to rouse in an emergency. Dependence may occur and the drugs may further disrupt sleep. They also increase the likelihood of sleep apnoea.

It is important to distinguish sleep disorders in the elderly from the normal sleep changes of later life and to classify them in order to plan appropriate treatment.

\section{Normal sleep}

Two distinct types of sleep can be identified by electroencephalograms (EEGs). These are rapid eye movement (REM) sleep and non-REM sleep. Table 1 summarises the EEG characteristics of normal sleep.

The physiological characteristics of REM and non-REM sleep differ. During REM sleep there is a predominance of sympathetic activity with ocular movements, loss of muscle tone, increases in systolic blood pressure, heart rate and respiratory rate accompanied by penile erection or increased vaginal blood flow (Snyder et al, 1964). Dreaming occurs principally during REM sleep. During non-REM sleep there is a preponderance of parasympathetic activity with the corresponding physiological effects.

\section{Sleep changes in late life}

Sleep undergoes a number of changes with increasing age and tends to exhibit more varied patterns. Total sleep time decreases with age, but the amount of time spent in bed increases with a greater proportion of time in bed spent awake (Hartford \& Samorajski, 1982). The latter may partly reflect increased spare time available to retired or bereaved people, because both employment and marriage have an organising influence on sleep-wake patterns and following retirement or bereavement these patterns may change. Older people may pursue a rather improvised sleep pattern as a perceived benefit of retirement, and, following a bereavement, a person may retire to bed earlier through boredom (Vaughn McCall, 1995).

Sleep onset takes longer with age: approximately $32 \%$ of elderly women and $15 \%$ of elderly men take over 30 minutes to fall asleep. Awakenings in the night are more frequent: over $50 \%$ of elderly people report more than 30 minutes of wakefulness after sleep onset. Overall, less time in bed is spent sleeping. Among 60- to 80-year-olds sleep time averages 6-6.5 hours per night with 7.5-8 hours spent in bed. Older people can be described as having an impaired capacity to maintain sleep (Bixler et al, 1984; Bachman, 1992). Daytime napping is common (Evans \& Rogers, 1994). 


\begin{tabular}{|c|c|c|c|c|}
\hline Sleep phase & Sleep stage & EEG findings & $\begin{array}{l}\text { Wave } \\
\text { characteristics }\end{array}$ & Changes with age \\
\hline \multirow[t]{5}{*}{ Non-REM } & $\begin{array}{l}\text { Stage } 0 \text { (drowsy, } \\
\text { eyes closed) }\end{array}$ & Alpha activity & $8-12 \mathrm{~Hz}$ & \\
\hline & Stage 1 & $\begin{array}{l}\text { Emergence of theta } \\
\text { waves, disappearance } \\
\text { of alpha waves }\end{array}$ & $3-7 \mathrm{~Hz}$ & $\begin{array}{l}\text { Increased proportion } \\
\text { of sleep }\end{array}$ \\
\hline & $\begin{array}{l}\text { Stage } 2 \\
\text { (deepening sleep) }\end{array}$ & $\begin{array}{l}\text { Further slowing } \\
\text { K-complexes } \\
\text { Sleep-spindles }\end{array}$ & $\begin{array}{l}2-7 \mathrm{~Hz} \\
\text { Single, high- } \\
\text { amplitude waves, } \\
\text { lasting }>0.5 \mathrm{~s} \\
\text { Runs of rapid } \\
(12-14 \mathrm{~Hz}) \text { waves } \\
\text { lasting }>0.5 \mathrm{~s}\end{array}$ & $\begin{array}{l}\text { Interspersed between } \\
\text { REM phases }\end{array}$ \\
\hline & $\begin{array}{l}\text { Stage } 3 \\
\text { (deep, slow- } \\
\text { wave sleep) }\end{array}$ & $\begin{array}{l}\text { Delta activity } \\
\text { (constituting } \\
20 \% \text { of total activity) }\end{array}$ & $0.5-2 \mathrm{~Hz}$ & $\begin{array}{l}\text { Decreased proportion } \\
\text { of sleep }\end{array}$ \\
\hline & $\begin{array}{l}\text { Stage } 4 \\
\text { (deep, slow- } \\
\text { wave sleep) }\end{array}$ & $\begin{array}{l}\text { Delta activity } \\
\text { (constituting } \\
50 \% \text { of total activity) }\end{array}$ & $0.5-2 \mathrm{~Hz}$ & $\begin{array}{l}\text { Decreased proportion } \\
\text { of sleep }\end{array}$ \\
\hline REM & & $\begin{array}{l}\text { Theta activity similar } \\
\text { to Stage } 1 \text { sleep but } \\
\text { with the addition of } \\
\text { sawtooth waves }\end{array}$ & $3-7 \mathrm{~Hz}$ & $\begin{array}{l}\text { Decreased proportion of } \\
\text { total sleep time but length } \\
\text { of REM phases remains } \\
\text { constant }\end{array}$ \\
\hline
\end{tabular}

Polysomnography is a technique which combines EEG monitoring during sleep with electro-oculography to study eye movements, electrocardiography, respiratory monitoring and the simultaneous monitoring of other physiological variables such as movement, temperature and penile tumescence. Polysomnographic studies have revealed electrophysiological changes with advancing age. There is a steady decrease in the proportion of Stage 3 and 4 slow-wave deep sleep (Karacan \& Williams, 1983). Stages 3 and 4 are almost absent by the age of 60 years. The first sleep cycle is predominantly Stage 4 and this decreases in length in old age. The proportion of Stage 1 sleep increases markedly. After the age of 70 years, men exhibit no Stage 4 sleep and only about $1 \%$ of sleep is Stage 3 . The change is less marked in women.

Changes in REM sleep are also evident in old age. After the age of 70 years, the proportion of REM sleep declines rapidly. In younger adults the length of each REM phase increases with each sleep cycle. In the elderly the length of REM phases remains constant and they are often interspersed with periods of Stage 2 non-REM sleep.

\section{Sleep disorders in the elderly}

More than 80 sleep disorders are classified in the international classification of the American Sleep Disorders Association (ASDA) (ASDA, 1997). They are divided into dyssomnias, parasomnias, medical and psychiatric sleep disorders and other proposed sleep disorders. Table 2 outlines the ASDA classification, with examples of sleep disorders in each category.

The ASDA criteria cover all types of sleep disorder, both organic and non-organic. In the ICD-10 classification (World Health Organization, 1992) only non-organic sleep disorders are classified under chapter V (mental and behavioural disorders; F-codes) where they are divided into dyssomnias and parasomnias (as per the ASDA criteria). Organic disorders are classified elsewhere in ICD10 (G-codes) under diseases of the nervous system. This division complicates the classification of sleep disorders using ICD-10. For example, in ICD-10, dyssomnias include only insomnia, hypersomnia 
and non-organic disorders of the sleep-wake schedule, whereas the ASDA criteria include such disorders as restless legs syndrome, narcolepsy and sleep apnoea, which are found under nervous system disorders in ICD-10 (G25, G47.4 and G47.3, respectively). Parasomnias in ICD-10, chapter V, include psychogenic sleep disorders such as sleepwalking, sleep terrors and nightmares, whereas the ASDA criteria for parasomnias include other disorders such as REM sleep behavioural disorder and paroxysmal nocturnal dystonia.

The ASDA criteria are practically useful and provide more detailed diagnostic criteria for sleep disorders than ICD-10. The ASDA classification is used below.

\section{Dyssomnias}

\section{Intrinsic sleep disorders}

The most common intrinsic dyssomnias in the elderly are primary snoring, sleep apnoea, restless legs syndrome and periodic movements of sleep.

\section{Primary snoring}

This is very common in the elderly. It occurs in up to $60 \%$ of men and $40 \%$ of women (Becker \& Jamieson,
1992) and is associated with an increase in mortality due to heart disease and hypertension. Distress and disruption to the sleeping partner of the sufferer may be considerable. Snoring may be the presenting symptom of sleep apnoea, but commonly occurs in isolation.

Management is conservative and may include weight loss, abstinence from night-time alcohol and sedatives and avoidance of supine sleeping positions. Sometimes nasal pathology may be involved, such as allergic rhinitis - referral to an ear, nose and throat clinic may be appropriate.

\section{Sleep apnoea}

This disorder is common in elderly people and is characterised by recurrent disordered breathing during sleep with apnoeas and hypopnoeas. In sleep apnoea syndrome apnoeas occur more frequently than five times per hour and last for 10 seconds or more (as defined by the ASDA). Studies have shown prevalence rates ranging from 26 to $73 \%$ of elderly populations (Fleury, 1992). The syndrome may be linked with upper respiratory tract obstruction or central respiratory inhibition (Karacan \& Williams, 1983). Obstructive sleep apnoea is more common in men, associated with obesity and characterised by loud snoring. It can result in insomnia, restless sleep, daytime sleepiness, daytime cognitive impairment, depression, nocturia and impotence (Wooten, 1992). Subjective complaints of nocturia may be the

\section{Table 2 Summary of the American Sleep Disorders Association classification of sleep disorders}

Dyssomnias

Intrinsic sleep disorders

Extrinsic sleep disorders

Circadian rhythm disorders

Parasomnias

Arousal disorders

Sleep-wake transition disorders

Parasomnias associated with REM sleep

Other parasomnias

Medical/psychiatric

sleep disorders

Associated with mental disorders

Associated with neurological disorders

Associated with medical disorders
Psychophysiological insomnia, obstructive sleep apnoea, restless legs syndrome, narcolepsy

Poor sleep hygiene, alcoholdependent sleep disorder

Time zone change syndrome, delayed sleep phase disorder

Sleep-walking, sleep terrors

Nocturnal leg cramps, rhythmic movement disorder

REM sleep behavioural disorder, nightmares

Nocturnal paroxysmal dystonia, nocturnal enuresis

Depression, anxiety, panic disorder

Dementia, Parkinson's disease, sleeprelated headaches

Nocturnal cardiac ischaemia, sleeprelated asthma

Menstrual-associated sleep disorder 
presenting complaint. Pressman et al (1996) showed that, in a population of elderly persons with nocturia, over $79 \%$ had sleep apnoea, periodic leg movements or snoring. Only about $5 \%$ correctly identified these as the cause of their waking. The rest misattributed their awakenings to nocturia, presumably because many of them felt the need to urinate upon waking. Sleep apnoea is also associated with increased mortality, mainly due to its contribution to congestive cardiac failure and cardiac arrhythmias. This may account for the high incidence of deaths of elderly people during sleep (Karacan \& Williams, 1983).

Mild sleep apnoea can be managed conservatively. More severe cases may require continuous positive airway pressure ventilation via a nasal mask during sleep or, more rarely, surgical intervention.

\section{Periodic movements of sleep and restless legs syndrome}

These disorders are reported by approximately $5 \%$ of elderly people but may be present in up to $33 \%$ (Becker \& Jamieson, 1992).

Periodic movements of sleep presents with repeated flexion of the lower leg muscles every 2040 seconds, resulting in brief arousals from sleep. It results in excessive daytime sleepiness and is often only found after sleep studies. The aetiology is uncertain and may be either spinal or central. In a small percentage of cases, the disorder is due to vitamin B12 or iron deficiency.

Restless legs syndrome may cause marked insomnia. It consists of uncomfortable sensations within the leg muscles which are relieved briefly by voluntary movements of the legs.

Management of both conditions is usually pharmacological. Periodic movements of sleep responds to combined slow-release carbidopa/ levodopa (50/200 mg tablet at bedtime; Becker \& Jamieson, 1992). Restless legs syndrome shows a response to bromocriptine at doses up to $5 \mathrm{mg}$ twice daily. Other medication has been suggested, including opiates, but their mode of action is not known. Caffeine, other stimulants and exercise should be avoided within a few hours of retiring.

\section{Psychophysiological insomnia}

Adjustment sleep disorders can lead to the presence of psychophysiological insomnia, which occurs in up to $3 \%$ of elderly people (Becker \& Jamieson, 1992). In this condition, there is a preoccupation with bedtime and sleep rituals, resulting in frustration when insomnia occurs. This leads to mental and physical arousal, resulting in further sleep impairment. The condition occurs once the sleep impairment secondary to the adjustment episode has abated.
Sufferers characteristically go to bed at set times, before they are sleepy, and lie awake for long periods. They may often describe feeling sleepy before going to bed, but become alert once they get there. These disorders are more likely to occur following a change in life circumstance, such as retirement or spousal bereavement leading to increased spare time, boredom and perhaps earlier bedtimes. More time is spent in bed awake and there is a tendency to sleep late to compensate.

Treatment with drugs is best avoided, and behavioural techniques such as stimulus control therapy and sleep restriction therapy are appropriate and effective.

Stimulus control therapy (Bootzin \& Perlis, 1992) is a useful technique when sleep onset is disrupted. The aim of treatment is to enable sleep onset within 10 minutes of going to bed. The patient is instructed to get out of bed if they are not asleep within 10 minutes and only return there once they feel sleepy again. This is repeated and sleep onset gradually occurs more quickly. Sleep restriction therapy (Spielman $e t$ al, 1987) is used where there are difficulties in maintaining sleep. A detailed sleep diary is kept for two weeks, recording estimates of time spent in bed and time spent sleeping. From these figures the approximate average number of hours sleep per night is calculated. The technique then requires the patient to spend only this amount of time in bed each night. For instance, if the average number of hours sleep per night is five hours, the patient is advised to not go to bed until 3 a.m. and to get up at 8 a.m. on a regular basis. Sleep will improve and as it does, the patient is allowed to retire to bed earlier, in 15-minute increments. This technique leads to deeper sleep with quicker onset and better sleep maintenance. It is demanding of the patient and high levels of motivation are required. Much support and encouragement needs to be given, particularly in the early stages of sleep restriction. Two-thirds of patients are said to improve by using this technique.

\section{Extrinsic sleep disorders}

There are 14 extrinsic sleep disorders listed in the ASDA classification. Inadequate sleep hygiene is the most common.

\section{Inadequate sleep hygiene}

Although classified by the ASDA separately, this overlaps with adjustment sleep disorder and psychophysiological insomnia. Its prevalence is unknown. Behaviours and practices are involved which are incompatible with sleep.

People who sleep poorly tend to have irregular hours of sleep, and to spend excessive time in bed 
trying to get to sleep. This is often followed by rising late to compensate for delayed sleep onset, and frequent naps during the day. Many other arousing behaviours can occur such as regular intake of caffeine (particularly late in the day), alcohol use, cigarettesmoking, working late and clock-watching. (Becker \& Jamieson, 1992). Inadequate sleep hygiene can also occur if the environment is not conducive to sleep. The bedroom may be noisy, too hot or too cold, or the patient may not feel secure there. Behaviour modification techniques to ensure good sleep hygiene are essential and can markedly improve sleep.

\section{Circadian rhythm disorders}

There are seven circadian rhythm disorders described in the ASDA classification and they have much in common. They include time zone change syndrome (jet-lag) and delayed sleep phase disorder. The most characteristic historical finding is that the total number of hours sleep per night is adequate, but the sleep phase is shifted so that sleep occurs at abnormal times. In the elderly the shift tends towards earlier circadian rhythms with earlier waking times. It has been suggested that these changes are due to a shift away from normal adult biphasic sleep-wake cycles towards the polyphasic pattern seen in neonates (Vitellio \& Prinz, 1990a). These changes may be precipitated by social and environmental changes such as loss of a spouse or retirement, and tend to be related to boredom or loss of routine. Studies have demonstrated that phase-advanced sleep patterns are also associated with institutionalisation (Middlekoop et al, 1994). Elderly people in care are also likely to be prescribed sedative hypnotic drugs, which may be relevant to sleep disorders in institutional settings.

Treatment of circadian rhythm disturbances is usually by behavioural techniques, including encouragement of gradually later bedtimes. Phototherapy has also been used to some effect.

\section{Parasomnias}

Parasomnias are involuntary behaviours occurring during sleep or during the sleep-wake transition. The ASDA classification describes a total of 24 parasomnias including arousal disorders, sleepwake transition disorders, REM sleep parasomnias and others (see Table 2). Non-REM sleep behaviour disorders such as sleep-walking and night terrors, which are the best known examples, tend to dissipate by young adulthood and are unusual in the elderly. If activity resembling sleep-walking or night terrors presents in the elderly an underlying central nervous system disorder should be suspected. Such behaviour in elders with a dementia is likely to represent episodes of nocturnal confusion and wandering (Kales et al, 1987). The REM sleep parasomnias are more important in older age groups.

\section{Parasomnias associated with REM sleep}

REM sleep behaviour disorder (RBD)

This condition is characterised by a history of vigorous or violent activity during sleep, often with vocalisations. It usually occurs during the middle and last third of the night, and may be associated with injuries due to jumping or falling out of bed during the episode. Sufferers may report vivid frightening dreams (Pressman et al, 1996; Chiu et al, 1997). The disorder is often misdiagnosed and its prevalence is unknown. It appears to be more common in older men. Just over $77 \%$ of people with RBD present with sleep-related injuries (Morfis et $a l, 1997)$ so careful assessment and diagnosis is necessary.

There are two forms of RBD, acute and chronic. The acute form can be precipitated by drugs such as tricyclic antidepressants, monoamine oxidase inhibitors and also by alcohol withdrawal. The chronic form is often idiopathic but may occur in neurological disorders such as Parkinson's disease, multiple sclerosis, cerebrovascular ischaemia, olivopontocerebellar degeneration, dementia and central nervous system neoplasms. Diagnosis involves careful history-taking and investigations such as EEGs and polysomnography. An EEG will help to distinguish RBD from nocturnal epilepsy and nocturnal confusional states in dementia. Polysomnography can help to identify abnormal movements during sleep associated with obstructive sleep apnoea.

The treatment of choice for RBD is clonazepam which is effective in up to $90 \%$ of cases (Chiu et al, 1997).

\section{Medical and psychiatric sleep disorders}

\section{Sleep disorders associated with mental disorders}

Adjustment sleep disorder

Adjustment sleep disorder is common in the elderly with a prevalence of about 35\% (Becker \& Jamieson, 
1992). It may follow psychosocial stressors such as death of a spouse, change of residence or physical illness. Sleep is disrupted because of difficulties with sleep onset. Concern and worry over not sleeping exacerbates the problem and can lead to psychophysiological insomnia which persists after the reaction to the stressor has subsided. Problems for the sufferer include daytime irritability, anxiety, slowing of cognition, lethargy and tearfulness.

Adjustment sleep disorder usually resolves with time. Psychological support and behavioural methods including restoration of proper sleep hygiene are useful. Hypnotics should be avoided if possible.

\section{Anxiety disorders}

Generalised anxiety disorder, panic disorder and obsessive-compulsive disorder are all associated with sleep disruption. Sleep onset difficulties are characteristic. Most elderly people with anxietyrelated sleep difficulties will have already had anxiety problems for many years and may be receiving drug treatments. New presentations of anxiety disorders in later life should be carefully assessed to rule out other causes of anxiety and sleep disorder, including depression, medical disorders, dementia, substance misuse and other sleep disorders.

Treatment involves psychological management of the anxiety disorder and behavioural management of any sleep disorder, with avoidance of benzodiazepines and other sedative hypnotics. If unavoidable, benzodiazepines may be prescribed in small doses for time-limited periods, preferably only up to three nights per week, in order to avoid tolerance and dependence.

\section{Mood disorder}

Sleep disturbances are intimately associated with affective disorders. In depression sleep maintenance is disturbed and early morning awakening is common. Sleep onset may be affected when anxiety symptoms are prominent. Daytime lethargy and psychomotor retardation may predispose to daytime napping or withdrawal to bed, which may further disrupt sleep. Cognitive impairment may be a presenting symptom of depressive illness in the elderly and may be exacerbated by sleep difficulties.

Conversely, sleep difficulties themselves may predispose to depressive illness. In one study of 1801 subjects over the age of 65 years $12 \%$ had significant insomnia (Ford \& Kamerow, 1989). After one year, those who still suffered from insomnia were up to 40 times more likely to have developed a depressive disorder. Reynolds et al (1993) suggest that sleep may be a key factor in promoting resilience after stressful life events in later life.
Significant changes in the architecture of sleep can be demonstrated by EEGs of sleep in depressive illnesses. Sleep continuity is disrupted. The time from onset of sleep to when REM sleep first occurs (REM latency) is shortened and there is a reduction in slow-wave sleep (Wooten, 1992). Among elderly people with depression REM latency is commonly less than 10 minutes. In addition, REM episodes are longer and constitute a greater proportion of sleep time. Severity of cognitive impairment appears to be related to the degree of sleep fragmentation caused by depression.

Antidepressant therapy will help sleep via its effect on the depressive disorder itself, but patients whose sleep disturbances are particularly troublesome or distressing may benefit from sedative antidepressants.

\section{Alcohol dependence}

Alcohol misuse is associated with sleep disturbance in the elderly and may be unsuspected. Alcohol may also be used for self-medication when there is an existing sleep disorder and can further disrupt sleep. It decreases REM sleep and decreases Stages 3 and 4 of non-REM sleep (Hartford \& Samorajski, 1982). Alcohol withdrawal also causes sleep disturbances. It is important to obtain a detailed alcohol history for anyone complaining of sleep difficulties.

\section{Sleep disorders associated with neurological disorders}

A number of neurological conditions are associated with significant sleep disturbance, including the dementias and Parkinson's disease.

\section{Dementia}

Sleep disorder is well recognised in dementias of all types. Increased awakenings are common, with night-time wandering, agitation and confusion (Becker \& Jamieson, 1992). These are a source of much distress to carers (Burns et al, 1990). Abnormalities occur in the sleep-wake patterns of these people, possibly secondary to degenerative brain changes in the neuronal pathways associated with initiation and maintenance of sleep (Vitellio \& Prinz, 1990a). The degree of disruption of sleep-wake patterns in Alzheimer's-type dementia correlates with the degree of cognitive impairment. There is a decrease in the proportion of time spent in bed, in slow-wave sleep time and in REM sleep time along with increased awakenings and loss of the normal sleep-wake cycle. Sleep apnoea is much more common in women with Alzheimer's disease than 
the general population, but there is no difference in men (Vitellio \& Prinz, 1990b).

Carers should be informed of the potential for sleep-wake changes in dementia and that severity is likely to increase with disease progression. Daytime napping should be discouraged where possible to help to consolidate night-time sleep. Use of sedative night medication may be necessary but carries the potential for detrimental adverse effects.

\section{Parkinson's disease}

Sleep disorders are common in Parkinson's disease sufferers. Partinen (1997) describes sleep disorders, with frequent awakenings and fragmentation of sleep, in up to $98 \%$ of people with idiopathic Parkinson's disease. Dystonia, nocturia, leg cramps, nightmares, back or limb pain, difficulty turning over and leg jerks may all be contributory. They are related to disease severity. Depression is also common in Parkinson's disease and may itself disrupt sleep.

Treatment may involve a variety of drugs. L-dopa at bedtime may be effective in alleviating nocturnal movements but may cause nightmares or hallucinations. Diphenhydramine may be useful for its mild hypnotic and mild anti-Parkinsonian effects. Nocturnal polyuria may be helped by intra-nasal desmopressin.

\section{Sleep disorders associated with other medical disorders}

Many medical disorders from which elderly people suffer may predispose to sleep disorder. Pain may be significant in many illnesses, including osteoarthritis, rheumatoid arthritis and neoplastic disease, and can have detrimental effects on sleep. Immobility is more common with increasing age and may further exacerbate sleep difficulties. Dyspnoea can cause difficulties in congestive cardiac failure, chronic obstructive airway disease and asthma. Gastric symptoms can cause recurrent awakenings. Cardiovascular disease is associated with increased mortality during sleep, particularly during episodes of REM sleep when there is irregular autonomic activity which increases the likelihood of arrhythmias and asystoles. Disruption of sleep is also common in angina sufferers: there is prolonged sleep latency, longer Stage 1 and 2 phases and less Stage 3 and 4 sleep with frequent arousals (Karacan \& Williams, 1983).

Apart from the physical effects of medical illnesses in the elderly, there can also be marked disruption of sleep due to the psychological stress associated with chronic or serious disease, which can lead to adjustment-type sleep disorders.
Visual impairment is common in later life and is another recognised cause of sleep disturbance (Karacan \& Williams, 1983), probably due to changes in the retino-pineal and retino-suprachiasmatic pathways which are important in the maintenance of circadian rhythms in lower species and possibly also humans.

Drug treatment of multiple illnesses in the elderly often results in polypharmacy: many drugs have sleep-disrupting properties.

\section{Drugs and sleep disorders}

Many commonly prescribed drugs have sleep-related side-effects, particularly in elderly people. Insomnia can be caused by anti-angina drugs such as nifedipine and diltiazem, by $\mathrm{H}_{2}$ antagonists such as ranitidine and by sedative hypnotics such as the benzodiazepines. The latter may appear to be a paradoxical effect, but benzodiazepines increase fast EEG activity and as such will reduce slow-wave sleep which is already decreased in older adults. Many non-psychotropic drugs can cause drowsiness or somnolence which interferes with sleep patterns, for example, digoxin, ranitidine, atenolol and various acetyl cholinesterase inhibitors.

Benzodiazepines are commonly prescribed for elderly people with sleep disturbances, but carry risks. Mortality is increased (Kripke et al, 1979), partly because cardiac arrhythmias are more likely in response to autonomic instability characteristic of increased REM-phase sleep. Falls, cognitive impairment and dependence are likely. Great care is necessary when prescribing this class of drug for older adults.

\section{Assessment}

Careful assessment of elderly people with sleep disorders is the foundation of diagnosis and treatment planning. A detailed history of the sleep disturbance will include information on sleep over a 24-hour cycle, including time of retiring to bed, timing of sleep, times of waking and information regarding any daytime sleep (Box 1). A collateral history from a bed partner helps in eliciting evidence of snoring, abnormal movements or behaviour problems during sleep. Medical and drug histories should be obtained with attention to symptoms such as nocturia which may be the presenting complaint of occult sleep disorders. A detailed psychiatric history including cognitive testing will help identify psychological disorders which may be precipitating 
Box 1. Essential components of a sleep history in an older person

\section{Subjective report of sleep disturbance}

Time of retiring to bed

Time of sleep onset

Number of awakenings and reasons for awakenings

Time to fall asleep again each time

Unusual symptoms during sleep

Time of finally waking up

Total sleep hours

How patient feels on waking

Any daytime napping

Times of exercise, if any

Caffeine intake (with times)

Alcohol intake (with times)

Level of pain at bedtime (if any)

Level of anxiety at bedtime

Medication

Activities before retiring to bed

sleep disturbance. A physical examination (including a full nervous system examination) is essential, followed by appropriate investigations, which may include EEG and referral for polysomnography.

\section{Conclusions}

Sleep disorders are common among older adults and result in impaired daytime functioning, impaired cognition, increased mortality and over-prescribing of hypnotics. Poor sleep is more than just another symptom of advancing age and can have significant effects on both the physical and mental health of the sufferer. Health professionals know little about sleep and the treatment of sleep disorders is therefore generally poor. Those who do not understand sleep disorders tend to treat them with hypnotic drugs and often do more harm than good.

Education of health care professionals in the assessment and treatment of sleep disorders is essential if these problems are to be avoided.

\section{References}

American Sleep Disorders Association (1997) International Classification of Sleep Disorders, Revised: Diagnostic and Coding Manual. Rochester, MN: ASDA.
Bachman, D. L. (1992) Sleep disorders with aging: evaluation and treatment. Geriatrics, 47, 53-61.

Becker, P. M. \& Jamieson, A. O. (1992) Common sleep disorders in the elderly: Diagnosis and treatment. Geriatrics, 47, 41-52.

Bixler, E. O., Kales, A., Jacoby, J. A., et al (1984) Nocturnal sleep and wakefulness: effects of age and sex in normal sleepers. International Journal of Neuroscience, 23, 33-42.

Bootzin, R. R. \& Perlis, M. L. (1992) Nonpharmacologic treatments of insomnia. Journal of Clinical Psychiatry, 53 (suppl. 6), 37-41.

Burns, A., Jacoby, R. \& Levy, R. (1990) Psychiatric phenomena in Alzheimer's disease. IV: Disorders of behaviour. British Journal of Psychiatry, 157, 86-94.

Chiu, H. F., Wing, Y. K., Chung, D. W., et al (1997) REM sleep behaviour disorder in the elderly. International Journal of Geriatric Psychiatry, 12, 888-891.

Evans, B. D. \& Rogers, A. E. (1994) 24-hour sleep/wake patterns in healthy elderly persons. Applied Nursing Research, 7, 75-83.

Fleury, B. (1992) Sleep apnoea syndrome in the elderly. Sleep, 15, S39-S41.

Ford, D. E. \& Kamerow, D. B. (1989) Epidemiologic study of sleep disturbances and psychiatric disorder. Journal of the American Medical Association, 262, 1479-1484.

Hartford, J. T. \& Samorajski, T. (1982) Alcoholism in the geriatric population. Journal of the American Geriatrics Society, 30, 18-24.

Kales, A., Soldatos, C. R. \& Kales, J. D. (1987) Sleep disorders: insomnia, sleepwalking, night terrors, nightmares and enuresis. Annals of Internal Medicine, 106, 582592.

Karacan, I. \& Williams, R. L. (1983) Sleep disorders in the elderly. American Family Physician, 27, 143-152.

Kripke, D. F., Simons, R. N., Garfinkel, L., et al (1979) Short and long sleep and sleeping pills: is increased mortality associated? Archives of General Psychiatry, 37, 103-116.

Middelkoop, H. A., Kerkhof, G. A., Smilde-van-den-Doel, D. A., et al (1994) Sleep and ageing: the effect of institutionalisation on subjective and objective characteristics of sleep. Age and Ageing, 23, 411-417.

Morfis, L., Schwarz, R. S. \& Cistulli, P. A. (1997) REM sleep behaviour disorder: a treatable cause of falls in elderly people. Age and Ageing, 26, 43-44.

Partinen, M. (1997) Sleep disorder related to Parkinson's disease. Journal of Neurology, 244 (suppl. 1), S3-S6.

Pressman, M. R., Figueroa, W. G., Kendrick-Mohamed, J., et al (1996) Nocturia: A rarely recognized symptom of sleep apnoea and other occult sleep disorders. Archives of Internal Medicine, 156, 545-550.

Reynolds, C. F., Hoch, C. C., Buysse, D. J., et al (1993) Sleep after spousal bereavement: a study of recovery from stress. Biological Psychiatry, 34, 791-797.

Snyder, F., Hobson, J. A., Morrison, D. F., et al (1964) Changes in respiration, heart rate, and systolic blood pressure in human sleep. Journal of Applied Physiology, 19, 417.

Spielman, A. J., Saskin, P. \& Thorpy, M. J., (1987) Treatment of chronic insomnia by restriction of time spent in bed. Sleep, 10, 45-56.

Vaughn McCall, W. (1995) Management of primary sleep disorders among elderly persons. Psychiatric Services, 46, 49-55.

Vitellio, M. V., \& Prinz, P. N. (1990a) Sleep and sleep disorders in normal aging. In Handbook of Sleep Disorders (ed. M. J. Thorpy), pp. 139-151. New York \& Basel: Marcel Dekker.

- \& - (1990b) Sleep/wake patterns and sleep disorders in Alzheimer's disease. In Handbook of Sleep Disorders (ed. M. J. Thorpy), pp. 703-718. New York \& Basel: Marcel Delker.

Wooten, V. (1992) Sleep disorders in geriatric patients. Clinics in Geriatric Medicine, 8, 427-439.

World Health Organization (1992) The ICD-10 Classification of Mental and Behavioural Disorders. Geneva: WHO. 


\section{Multiple choice questions}

1. In elderly people:

a total sleep time increases with age

b slow-wave sleep is almost absent by the age of 60 years

c the proportion of REM sleep increases with age

d sleep EEG changes are more prominent in males

e the length of each REM phase during each successive sleep cycle remains constant.

2. Sleep apnoea syndrome:

$a$ is classified under mental and behavioural disorders in ICD-10

b in the ASDA classification, is classified as a sleep disorder associated with medical disorders

c is more common in men than women

d can be effectively managed using continuous positive airway pressure ventilation

e is associated with daytime cognitive impairment.

3. Pharmacological treatments are the treatment of choice in:
a REM sleep behavioural disorder
b restless legs syndrome
c nocturnal movements in Parkinson's disease
d psychophysiological insomnia
e inadequate sleep hygiene.

4. In drug treatments in the elderly:

a benzodiazepines increase the proportion of deep slow-wave sleep

b ranitidine can cause insomnia

c mortality is increased with sedative hypnotic drugs

d atenolol can cause drowsiness

e anti-Parkinsonian drugs are contra-indicated in periodic movements of sleep.

5. In behavioural treatments of sleep disorders:

a sleep restriction therapy is not useful where maintenance of sleep is a problem

b exercise shortly before retiring should be discouraged

c loss of weight can be helpful in primary snoring

d use of small quantities of alcohol to assist with night sedation is advised

e it is useful to obtain a diary of the patient's sleep patterns.

\section{MCQ answers}

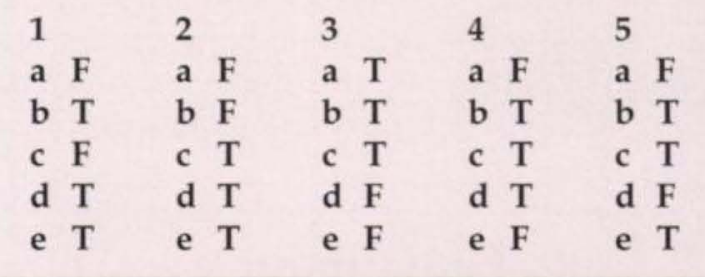

